\title{
Mechanical strain promotes osteogenic differentiation of bone mesenchymal stem cells from ovariectomized rats via the phosphoinositide 3-kinase/Akt signaling pathway
}

\author{
NINGJUAN OUYANG ${ }^{1}$, PENG ZHANG ${ }^{2}$, RUNQING FU ${ }^{1}$, GUOFANG SHEN ${ }^{3}$, LINGYONG JIANG ${ }^{1}$ and BING FANG ${ }^{1}$ \\ ${ }^{1}$ Center of Craniofacial Orthodontics, Department of Oral and Craniomaxillofacial Surgery; ${ }^{2}$ The Second Dental Center; \\ ${ }^{3}$ Department of Oral and Craniomaxillofacial Surgery, Shanghai Ninth People's Hospital, Shanghai Jiao Tong University School of \\ Medicine, Shanghai Key Laboratory of Stomatology, Shanghai 200011, P.R. China
}

Received April 26, 2017; Accepted September 7, 2017

DOI: $10.3892 / \mathrm{mmr} .2017 .8030$

\begin{abstract}
Osteoporosis has become an overwhelming public health problem worldwide. As an elementary physiological factor to regulate bone formation and regeneration, mechanical strain may be used as a non-invasive intervention in osteoporosis prevention and treatment. However, little is known regarding the underlying mechanism. The aim of the current study was to investigate the effect of continuous mechanical strain (CMS) on osteogenic differentiation of bone mesenchymal stem cells (BMSCs) from ovariectomized rats (OVX BMSCs). In addition, involvement of the phosphatidylinositol 3-kinase (PI3K)/Akt signaling pathway in biomechanical signal transduction and its function were evaluated. The results demonstrated that OVX BMSCs subjected to CMS exhibited higher alkaline phosphatase (ALP) activity and deeper staining at 24 and $48 \mathrm{~h}$. In addition, CMS upregulated the mRNA expression levels of ALP, collagen type I, runt related transcription factor 2 (Runx2), as well as the protein expression level of Runx 2 in a time-dependent manner. The PI3K/Akt signaling pathway was rapidly activated by CMS, with its phosphorylation level reaching its maximum in a short duration and a large quantity of phosphorylated-Akt remaining in the nucleus. Pre-treatment with a selective blocker significantly blocked the strain-induced activation of PI3K/Akt and reduced the commitment of OVX BMSCs into osteoblasts, demonstrating a dominated regulative effect
\end{abstract}

Correspondence to: Dr Bing Fang or Dr Lingyong Jiang, Center of Craniofacial Orthodontics, Department of Oral and Craniomaxillofacial Surgery, Shanghai Ninth People's Hospital, Shanghai Jiao Tong University School of Medicine, Shanghai Key Laboratory of Stomatology, 639 Zhizaoju Road, Shanghai 200011, P.R. China

E-mail: fangbing@sjtu.edu.cn

E-mail: jly117@sina.com

Key words: mechanical strain, osteoporosis, bone mesenchymal stem cells, osteogenic differentiation, phosphoinositide 3-kinase/Akt signaling pathways of PI3K/Akt signaling in strain-induced osteogenesis. These results indicated that CMS induced the early differentiation of OVX BMSCs towards an osteogenic phenotype by activating the PI3K/Akt signaling pathway.

\section{Introduction}

Osteoporosis, a condition characterized by a reduction in bone mass and strength, is associated with increased risks for fracture. It has become an overwhelming public health problem worldwide, particularly in postmenopausal women (1). The average bone loss during the five years around the menopause or perimenopause reaches $15 \%$, and postmenopausal women with low bone density are very likely to be predisposed to fractures $(2,3)$. Currently, pharmacological interventions for osteoporosis are classified into antiresorptive agents that prevent bone resorption, such as hormone replacement therapy, bisphosphonates and denosumab, and anabolic agents, which help with formation of new bones, including strontium and teriparatide. However, the efficacy of certain drugs is limited by perceived intolerance and long-term adverse events (4-6). Mechanical strain is known as the elementary physiological factor that regulates bone formation and regeneration, as well as maintaining the integrity of bone structure and function. Evidence indicated that physical exercise may improve skeletal resistance to bone fracture, and delay the progress of osteoporosis by enhancing bone mass and strength $(7,8)$. Therefore, physical activity may be used as a non-invasive intervention in osteoporosis prevention and treatment. However, little is known about the specific mechanism that regulates bone remodeling in osteoporosis.

Bone mesenchymal stem cells (BMSCs) are force-sensitive cells capable of detecting, transducing and responding to an extracellular stimulus, and thus differentiate into multiple cell lineages $(9,10)$. Evidence indicates that the osteogenic ability of BMSCs is key in bone remodeling. The alterations in BMSCs associated with estrogen reduction may result in the attenuated regenerative ability of bone, which consequently results in osteoporosis. Additionally, BMSCs are proposed to be of great importance in the response of bone to mechanical stimulation (11-13). However, few studies focused on the signaling 
pathway involved in bio-mechanical transduction of BMSCs from ovariectomized rats (OVX BMSCs) in vitro. Thus, such studies regarding the effect of mechanical strain on OVX BMSCs may elucidate the mechanism of bone remodeling in osteoporosis.

It is well known that the signal transduction initiated by external chemical or mechanical stimulation is important in regulating bone development (14). The phosphatidylinositol 3-kinase (PI3K)/Akt signaling pathway is one of the most common signaling pathways that has been identified to be implicated in BMSC proliferation and differentiation by modulating the transcriptional activity of downstream genes (15). In addition, there is substantial evidence that the PI3K/Akt signaling pathway is essential for human and murine MSC osteogenesis in vitro $(16,17)$. However, whether the PI3K/Akt signaling pathways is involved in the response of OVX BMSCs to mechanical strain has not, to the best of our knowledge, been thoroughly investigated. Therefore, by adopting an FX-4000T ${ }^{\mathrm{TM}}$ Tension Plus ${ }^{\mathrm{TM}}$ system, the mechanical environment of BMSCs in vivo was mimicked in the present study. Furthermore, the effect of continuous mechanical strain (CMS) on osteogenic differentiation of OVX BMSCs, and the involvement and function of the PI3K/Akt signaling pathway in biomechanical signal transduction were investigated.

\section{Materials and methods}

Animals and cell culture. The current study was conducted in accordance with the regional Ethics Committee guidelines. Sixty female Sprague-Dawley rats (age, 6 weeks), weighing an average of $200 \mathrm{~g}$, were obtained from Shanghai SLAC Experimental Animal Center (Shanghai, China). The animals underwent surgical ovariectomy according to FDA guidelines (18). The rats were then housed separately in a temperature-controlled room at $21^{\circ} \mathrm{C}$ with relative humidity at $60 \%$ under a 12-h light/dark cycle. Then, 12 weeks after ovariectomy, all rats were sacrificed. The humeri and tibiae were isolated from the OVX rats. The bone marrow was flushed out using Dulbecco's modified Eagle's essential medium (HyClone; GE Healthcare Life Sciences, Logan, UT, USA), supplemented with $100 \mathrm{U} / \mathrm{ml}$ penicillin, and $100 \mu \mathrm{g} / \mathrm{ml}$ streptomycin (Hyclone; GE Healthcare Life Sciences). Non-adherent cells were removed by replacing the medium after $72 \mathrm{~h}$ and it was subsequently refreshed every 3 days. On reaching 70-80\% confluence, the cells were trypsinized with $10 \%$ trypsin-EDTA (Hyclone; GE Healthcare Life Sciences) and passaged. OVX BMSCs from passages 2 to 5 were used during the experiments.

Application of CMS. CMS of $10 \%$ elongation at a frequency of $1 \mathrm{~Hz}$ was applied using an FX-4000T ${ }^{\mathrm{TM}}$ Flexercell Tension Plus $^{\mathrm{TM}}$ unit (Flexcell International Corp., Burlington, NC, USA). BMSCs were plated on Flexcell 6-well silicone rubber plates at a density of $2 \times 10^{4} / \mathrm{cm}^{2}$. After $24-48 \mathrm{~h}$ incubation, the cells had attached and reached $\sim 80 \%$ confluence. The BMSCs were then subjected to CMS for $48 \mathrm{~h}$.

Alkaline phosphatase $(A L P)$ staining and relative $A L P$ activity detection. The presence of ALP in the cell layers was assessed according to the manufacturer's protocol of the 5-bromo-4-chloro-3-indolyl-phosphate/nitro blue tetrazolium Alkaline Phosphatase Color Development kit (cat. no. C3206; Beyotime Institute of Biotechnology, Haimen, China) and described as follows. The OVX BMSCs were rinsed with phosphate-buffered saline (PBS) three times and fixed with $4 \%$ paraformaldehyde for $15 \mathrm{~min}$. Coloration was then assessed and observed with a digital camera (Eclipse TS100; Nikon Corporation, Tokyo, Japan). The relative ALP activity was detected according to the manufacturer's protocol with the Alkaline Phosphatase Assay kit (Beyotime Institute of Biotechnology). After exposing to CMS for 24 and $48 \mathrm{~h}$, samples from all groups were washed twice with double-distilled water and lysed via sonification. Cell lysates were incubated with p-nitrophenol phosphate (Beyotime Institute of Biotechnology) at $37^{\circ} \mathrm{C}$ for $1 \mathrm{~h}$. The enzymatic reaction was stopped using $1 \mathrm{M}$ sodium hydroxide and absorbance was measured at a wavelength of $405 \mathrm{~nm}$.

Reverse transcription-quantitative polymerase chain reaction $(R T-q P C R)$. Total RNA of the cells was isolated using TRIzol reagent (Invitrogen; Thermo Fisher Scientific, Inc., Waltham, MA, USA) according to the manufacturer's recommended protocol. The RNA concentrations were determined using a NanoDrop spectrophotometer (Thermo Fisher Scientific, Inc.) and cDNA was synthesized using a cDNA Synthesis Reverse Transcription kit (cat. no. RR037A; Takara Biotechnology Co., Ltd., Dalian, China). qPCR was performed using a Light-Cycler system with SYBR Premix Ex Taq ${ }^{\mathrm{TM}}$ (RR420A, Takara Biotechnology Co., Ltd.), according to the manufacturer's protocol. The conditions of the qPCR were as follows: Denaturation at $95^{\circ} \mathrm{C}$ for $10 \mathrm{sec}$, and 50 cycles of $95^{\circ} \mathrm{C}$ for $10 \mathrm{sec}$ and $60^{\circ} \mathrm{C}$ for $30 \mathrm{sec}$, with a final dissociation stage $\left(95^{\circ} \mathrm{C}\right.$ for $5 \mathrm{~min}$ ) to complete the amplification procedure. $\beta$-actin served as an internal control. The data were analyzed using comparative $\mathrm{C}_{\mathrm{q}}\left(2^{-\Delta \Delta \mathrm{Cq}}\right)$ method and expressed as a fold-change respective to the control (19). Each sample was analyzed in triplicate. The primer sequences used in the current study are presented in Table I.

Western blotting. The cells were lysed on ice for $30 \mathrm{~min}$ in SDS lysis buffer (Beyotime Institute of Biotechnology) supplemented with protease inhibitors. For western blot analysis, $20 \mu \mathrm{g}$ sample was resolved on a $10 \%$ SDS-PAGE gel and electro-transferred onto nitrocellulose membranes with a constant voltage of $90 \mathrm{~V}$ and duration of $70 \mathrm{~min}$ (Whatman, GE Healthcare Life Sciences). The following primary antibodies were used: Anti-runt related transcription factor 2 (Runx2; cat. no. 12256; 1:1,000; Cell Signaling Technology, Inc., Danvers, MA, USA); anti-Akt (cat. no. ab8805; 1:1,000; Abcam, Cambridge, MA, USA) and anti-p-Akt (cat. no. ab38449; 1:1,000; Abcam). For the normalization of protein loading, a GAPDH antibody (cat. no. 5174; Cell Signaling Technology, Inc.) was used at a dilution of 1:2,000. Horseradish peroxidase-conjugated secondary antibodies were used at a dilution of 1:5,000 (cat. no. ab6721; Abcam). The antigen-antibody complexes were visualized using an Enhanced Chemiluminescence Detection system (EMD Millipore, Billerica, MA), according to the manufacturer's protocols. Protein band intensities on the scanned films were compared to their respective controls using Alpha Image software. 
Table I. Reverse transcription-quantitative polymerase chain reaction primer sequences for target genes.

\begin{tabular}{lll}
\hline Gene & \multicolumn{1}{c}{ Forward (5'-3') } & \multicolumn{1}{c}{ Reverse $\left(5^{\prime}-3^{\prime}\right)$} \\
\hline$\beta$-actin & CACCCGCGAGTACAACCTTC & CCCATACCCACCATCACACC \\
Alkaline phosphatase & TATGTCTGGAACCGCACTGAAC & CACTAGCAAGAAGAAGCCTTTGG \\
Type I collagen & CAGGCTGGTGTGATGGGATT & CCAAGGTCTCCAGGAACACC \\
Runt related transcription factor 2 & ATCCAGCCACCTTCACTTACACC & GGGACCATTGGGAACTGATAGG
\end{tabular}

Inhibition of the PI3K/Akt signaling pathway. In order to assess the role of the PI3K/Akt signaling pathway in the strain-induced differentiation of OVX BMSCs, the selective inhibitor, LY294002 was used. Preliminary experiments indicated that the optimum concentration of LY294002 was $10 \mu \mathrm{M}$. Cells were pre-treated with inhibitors for $1 \mathrm{~h}$ prior to application of the strain stimulus, and they were present during the entire strain application.

Immunofluorescence analysis. Subsequent to mechanical loading, cells were fixed with $4 \%$ paraformaldehyde for $10 \mathrm{~min}$, then washed with PBS and incubated in $0.1 \%$ Triton $\mathrm{X}-100$ for $15 \mathrm{~min}$ at room temperature and then blocked with $5 \%$ bovine serum albumin for $1 \mathrm{~h}$ at room temperature. The prepared samples were incubated overnight at $4^{\circ} \mathrm{C}$ with rabbit monoclonal anti-phosphorylated (p)-Akt (Ser473; dilution, 1:300) or rabbit monoclonal anti-Akt (dilution, 1:300) that were obtained from Cell Signaling Technology, Inc., and detected with Alexa 594 conjugate (dilution, 1:200; Thermo Fisher Scientific, Inc.) at room temperature for $2 \mathrm{~h}$. Nuclei were labeled with $1 \mathrm{mg} / \mathrm{ml}$ Hoechst for $10 \mathrm{~min}$ at room temperature (Roche Diagnostics, Basel, Switzerland). Slides were examined under an Olympus IX71 fluorescent microscope. At least three overview images were obtained from three independent experiments.

Statistical analysis. All experiments were performed a minimum of three times and data are expressed as means \pm standard deviation. Differences between two groups were identified using unpaired t-tests. Significant differences between the non-load and multiple stretch groups were determined using a one-way analysis of variance followed by the Least Significant Difference post hoc test. $\mathrm{P}<0.05$ was considered to indicate a statistically significant difference.

\section{Results}

Effect of CMS on osteogenic differentiation of OVX BMSCs. After exposure to CMS, OVX BMSCs demonstrated higher ALP activity and deeper staining at 24 and $48 \mathrm{~h}$ when compared with the non-loaded OVX BMSC group (Fig. 1A and B). CMS upregulated the mRNA expression levels of osteogenesis-associated markers of OVX BMSCs, ALP, type I collagen (COL I) and Runx2, as they began to increase significantly at 4 or $6 \mathrm{~h}$ after exposure to CMS, and reached to a peak value at 24 or $48 \mathrm{~h}$ (Fig. 1C). Additionally, the protein expression level of Runx 2 was elevated in a time-dependent manner in OVX BMSCs compared with the non-loaded group, with a significant increase at 4 and $6 \mathrm{~h}$ (Fig. 1D and E).
Effects of CMS on induction of the PI3K/Akt signaling pathway. The activation time course of the PI3K/Akt signaling pathway was investigated in OVX BMSCs subjected to CMS. As demonstrated in Fig. 2A and B, Akt was significantly phosphorylated soon after the onset of stimulation and peaked at $15 \mathrm{~min}$. The phosphorylation level subsequently declined gradually, but remained higher than the non-loaded group at $30 \mathrm{~min}$. After $1 \mathrm{~h}$ of loading, the levels of p-Akt returned almost to baseline or were lower than the control group. The cellular localization of Akt and p-Akt was also examined by immunofluorescence analysis (Fig. 2C and D) and the nuclei were co-stained by Hoechst. Following CMS stimulation, Akt staining was performed with Akt antibodies, and observed in the cytoplasm and the nucleus at 1 and $4 \mathrm{~h}$. p-Akt staining was more strongly expressed in the nucleus than in the cytoplasm at $1 \mathrm{~h}$, and became markedly weaker at $4 \mathrm{~h}$.

Effects of a PI3K/Akt inhibitor on CMS-induced OVX BMSCs. The above-mentioned findings indicate the activation of the PI3K/Akt signaling pathway by CMS. After demonstrating that $\mathrm{PI} 3 \mathrm{~K} / \mathrm{Akt}$ may be involved in the mechanotransduction of CMS, its function in CMS-induced osteogenesis of OVX BMSCs was further investigated in the current study via pharmacological inhibition. LY294002 was used to block the activation of p-Akt in OVX BMSCs. Fig. 3A and B demonstrated that pre-treatment with Akt-specific inhibitor significantly blocked the phosphorylation of Akt and had no cytotoxic effect on the cells (data not shown). Immunofluorescence analysis of p-Akt co-confirmed that the activation of p-Akt was blocked by LY294002 treatment as p-Akt were predominantly stained in the cytoplasm (Fig. 3C and D).

Effects of a PI3K/Akt inhibitor on osteogenic differentiation of OVX BMSCs. Subsequently, CMS-induced osteogenesis of OVX BMSCs was assessed using a PI3K/Akt inhibitor. Pretreatment with LY294002 inhibited CMS-stimulated ALP activity (Fig. 4A and B). Furthermore, the CMS-induced mRNA expression levels of ALP, Col I and Runx2 were significantly repressed at 24 and 48 h (Fig. 4C). Similarly, as presented in Fig. 4D and E, the CMS-induced Runx2 protein expression level was attenuated by LY294002. These results indicate that the PI3K/Akt signaling pathway is responsible for the CMS-induced osteogenesis of OVX BMSCs.

\section{Discussion}

Recent studies have demonstrated that mechanical stimuli are essential for the differentiation of stem cells into different lineages. Lack of mechanical stress significantly attenuates 
A
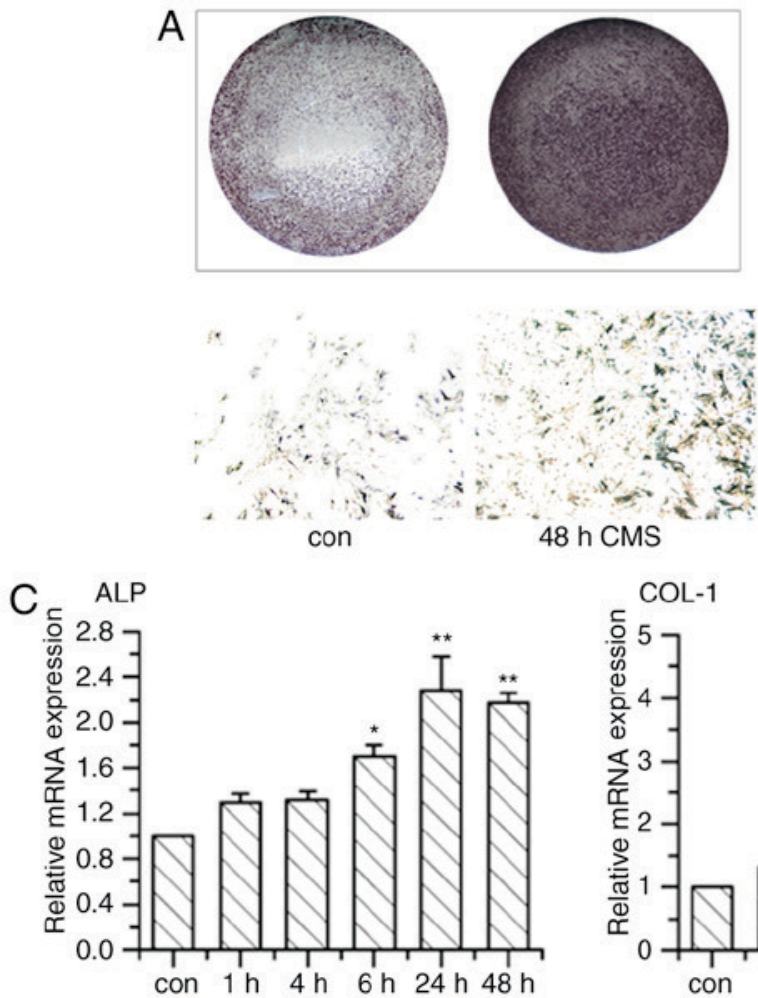

B

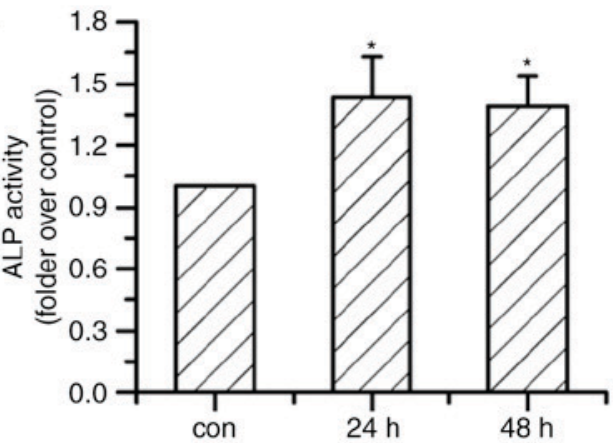

D
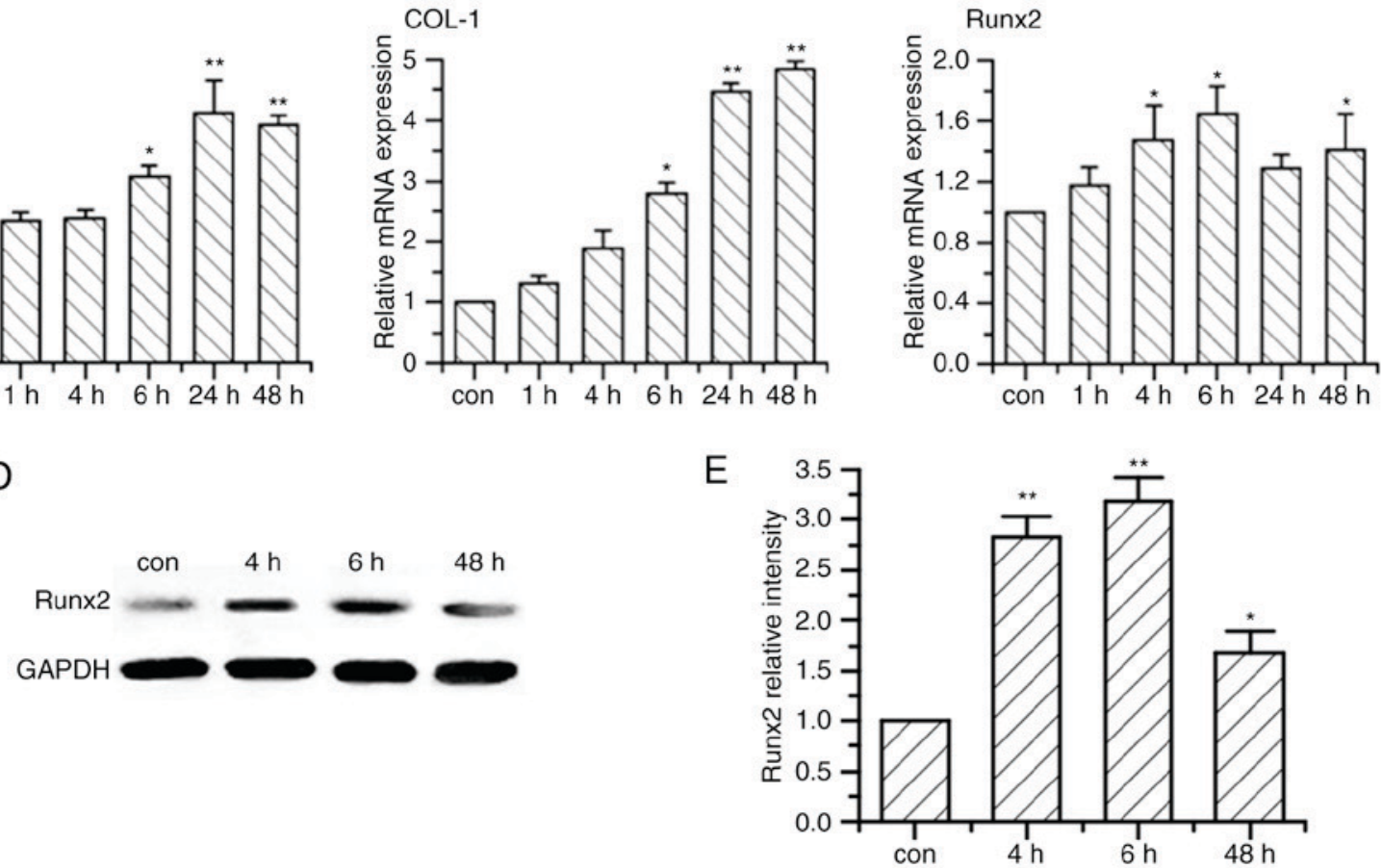

Figure 1. Effects of CMS on the osteogenic differentiation of OVX BMSCs. (A) ALP staining at $48 \mathrm{~h}$ vs. non-loaded control group (magnification, $\mathrm{x} 200$ ) and (B) ALP activity changes at 24 and $48 \mathrm{~h}$ vs. non-loaded control group. (C) mRNA changes of specific osteoblastic genes of OVX BMSCs at different time points following CMS. (D and E) Changes of Runx2 protein expression level following CMS. Each experiment was performed three separate times and the results are presented as means \pm standard deviation. ${ }^{*} \mathrm{P}<0.05$ and ${ }^{* *} \mathrm{P}<0.01$ vs. non-loaded control group of OVX BMSCs. CMS, continuous mechanical strain; BMSCs, bone mesenchymal stem cells; OVX BMSC, ovariectomized rat BMSC; ALP, alkaline phosphatase; Runx2, runt related transcription factor 2; COL I, type I collagen; con, control group.

the differentiating capability of BMSCs into osteoblasts, which may lead to disuse osteoporosis $(20,21)$. Characterized by decreased bone strength, osteoporosis is a chronic disease that easily predisposes individuals to fractures (22). As BMSCs are the progenitor cells of osteoblast cells, they are crucial in bone remodeling $(23,24)$. The current study was designed to evaluate the effects and specific underlying mechanism of CMS on the osteogenic differentiation of OVX BMSCs, with the aim of improving treatment strategies for osteoporosis.

BMSCs from osteoporosis patients exhibited longer population doubling duration. In addition, ovariectomy alters the synthesis of mineralized matrix and gene expression markers associated with osteogenic differentiation in BMSCs, and thus results in the reduction of the osteogenic potential $(25,26)$. Although our previous studies indicated that the ability of osteogenic lineage commitment of OVX BMSCs was weaker than sham BMSCs under the exposure of intermittent mechanical strain; the current study demonstrated that OVX BMSCs exposed to CMS underwent osteoblastic differentiation when compared with non-loaded OVX BMSCs (27). ALP activity and expression levels serve as indicators of osteoblastic activity. Extracellular matrix molecules, such as COL I, are considered to be of great importance in osteoblast proliferation and differentiation. Additionally, Runx 2 has been shown to be significant in regulating osteogenic differentiation (28). In the current study, the mRNA expression levels of ALP, COL I and Runx2 were enhanced in OVX BMSCs. Furthermore, OVX BMSCs subjected to CMS demonstrated higher ALP activities and deeper staining at 24 and $48 \mathrm{~h}$ when compared with the non-loaded OVX BMSC group. In addition, the protein expression level of Runx 2 was increased at 4 and $6 \mathrm{~h}$. These results demonstrated that OVX BMSCs underwent osteoblastic differentiation due to CMS. 

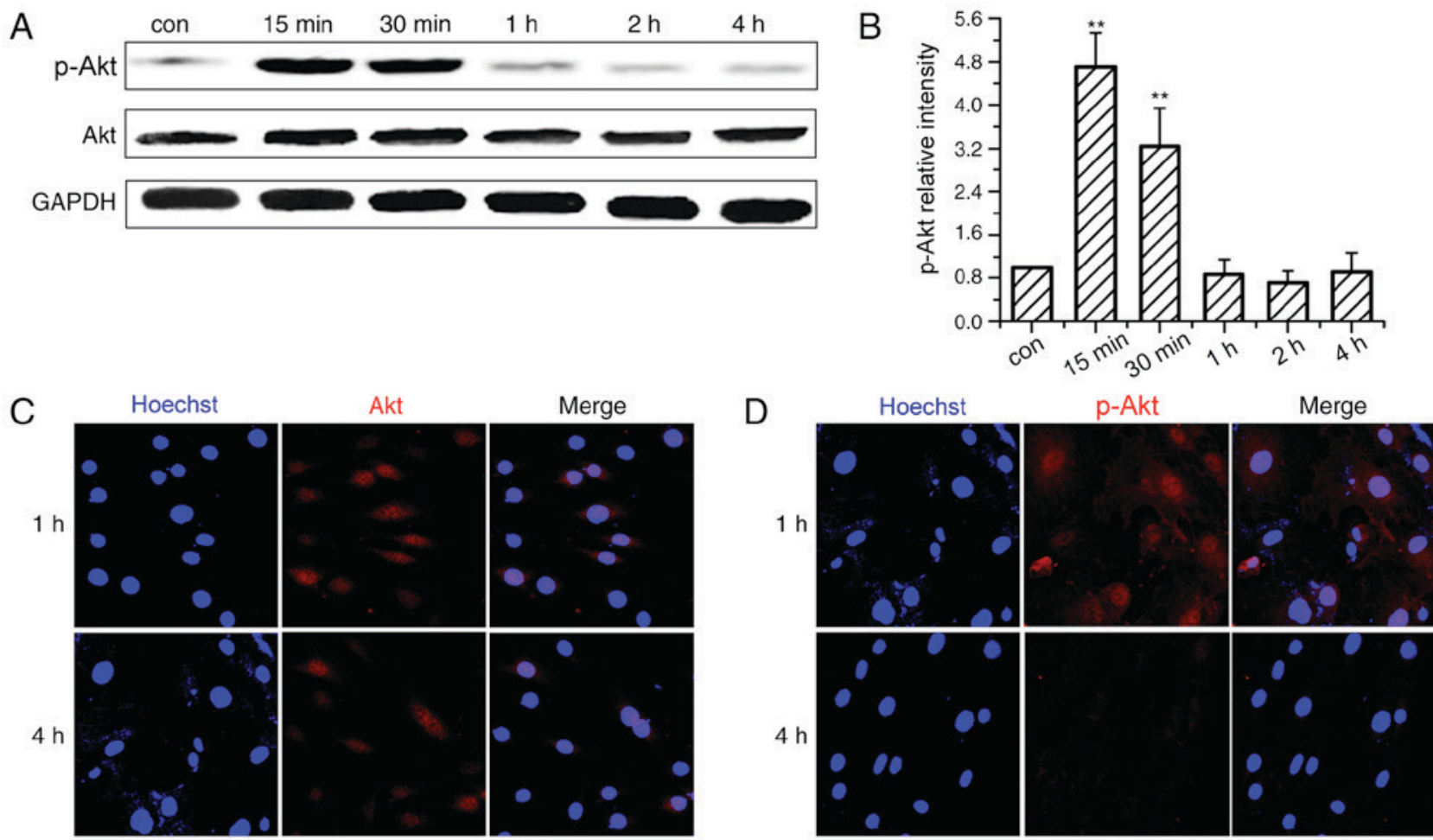

Figure 2. Effects of CMS on induction of the phosphatidylinositol 3-kinase/Akt signaling pathway. (A) Phosphorylation of Akt was examined by western blotting after CMS at the indicated time points. (B) Phosphorylated protein expression levels were normalized to their respective total protein levels. Immunofluorescent localization of (C) Akt and (D) p-Akt in ovariectomized rat bone mesenchymal stem cells subsequent to CMS (magnification, x200). Akt and p-Akt were labeled with an Alexa 594-conjugated antibody (red) and nuclei were counterstained with Hoechst (blue). The images demonstrate a typical example after 1 and $4 \mathrm{~h}$ of CMS. Values are presented as means \pm standard deviation $(\mathrm{n}=3)$. ${ }^{* *} \mathrm{P}<0.01 \mathrm{vs.}$. con group. CMS, continuous mechanical strain; $\mathrm{p}-$, phosphorylated; con, control group.

A

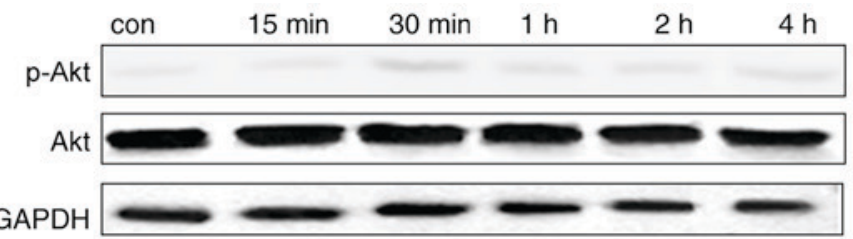

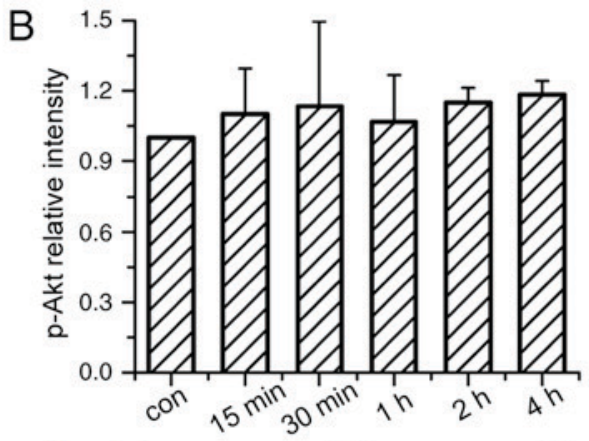
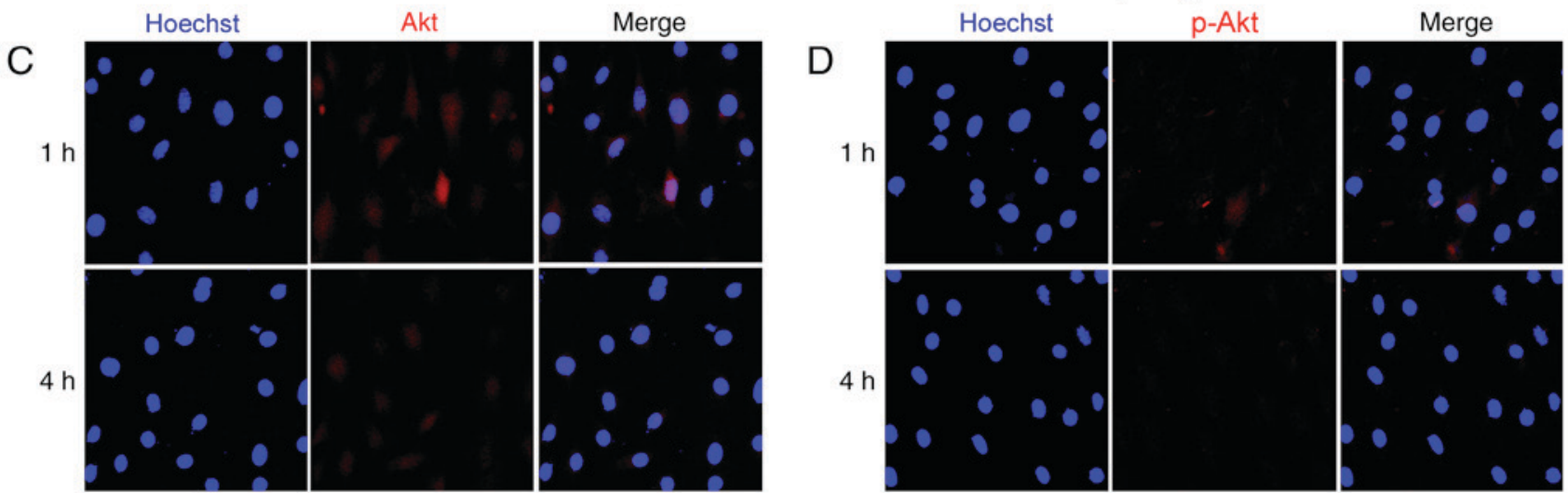

Figure 3. Involvement of a phosphatidylinositol 3-kinase/Akt inhibitor in OVX BMSCs under CMS. (A) Protein expression levels of Akt and p-Akt in OVX BMSCs treated with LY294002 were visualized by western blotting at the indicated time points. (B) Phosphorylated protein expression levels were normalized to their respective total protein levels. Immunofluorescent localization of (C) Akt and (D) p-Akt in OVX BMSCs upon CMS with the elective inhibitor, LY294002 (magnification, x200). The Akt and p-Akt was labeled with an Alexa 594-conjugated antibody (red), and nuclei were counterstained with Hoechst (blue). The images demonstrate a typical example after 1 and $4 \mathrm{~h}$ of CMS. Values were obtained from three individual experiments and presented as means \pm standard deviation ( $\mathrm{n}=3$ ). BMSCs, bone mesenchymal stem cells; OVX BMSC, ovariectomized rat BMSC; CMS, continuous mechanical strain; $\mathrm{p}$-, phosphorylated. 
A
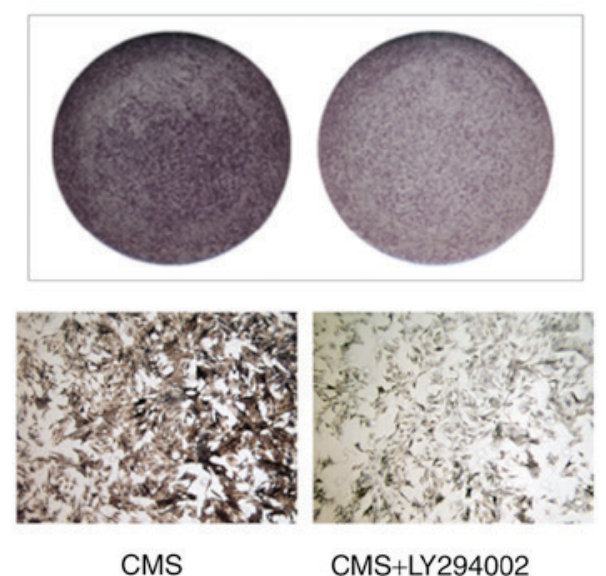

B

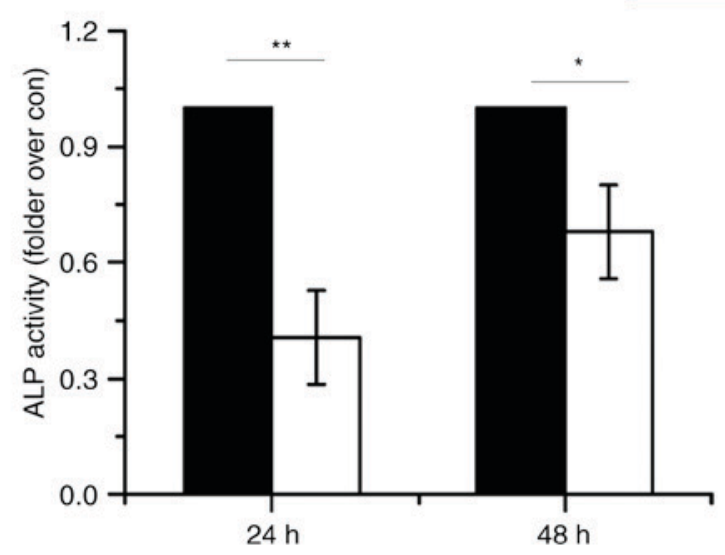

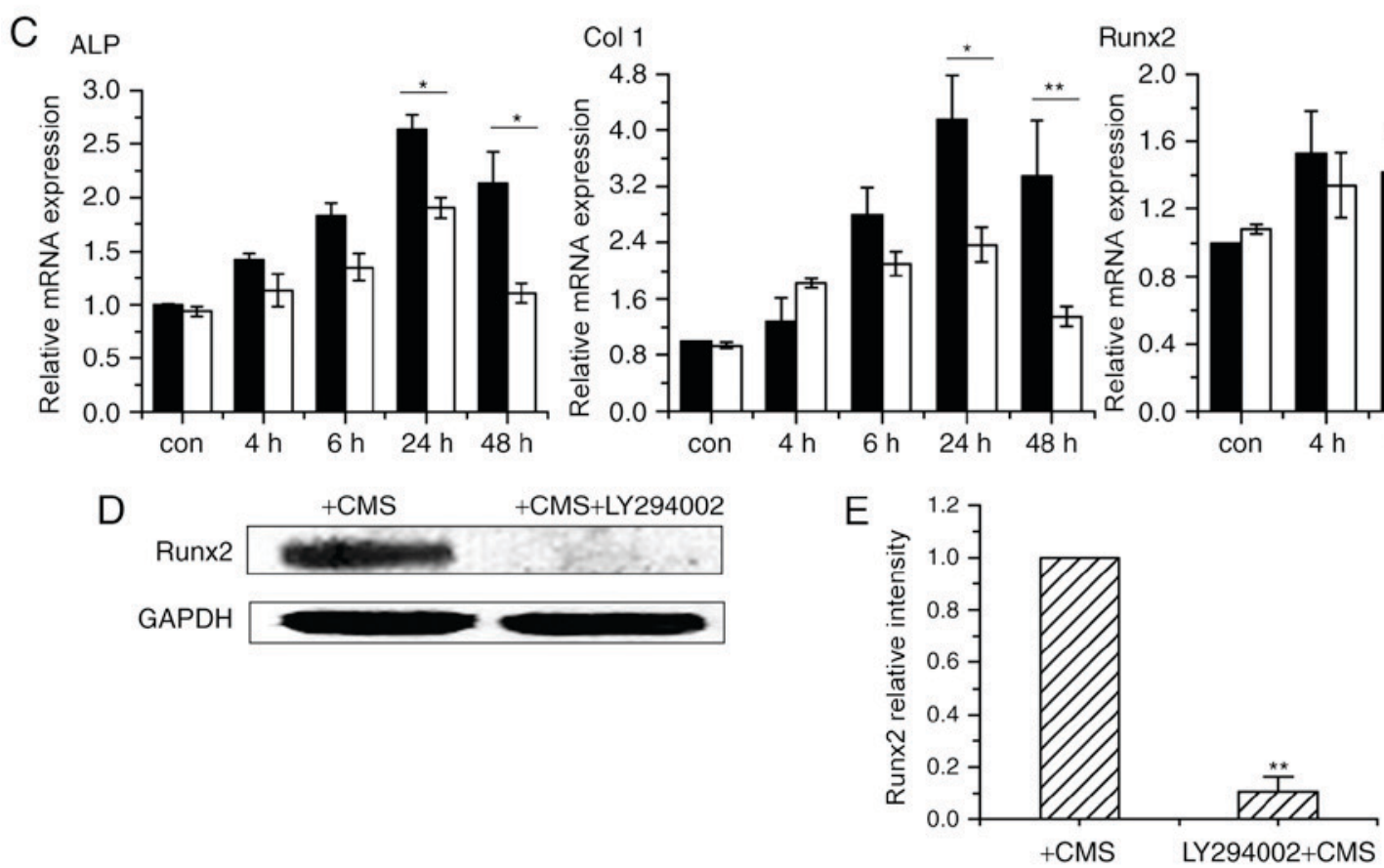

Figure 4. Effects of phosphatidylinositol 3-kinase/Akt signaling on osteogenic differentiation of OVX BMSCs following application of CMS. (A) Effects of an Akt-specific inhibitor on ALP staining at $48 \mathrm{~h}$ (magnification, x200) and (B) ALP activity at 24 and $48 \mathrm{~h}$. (C) Changes of CMS-induced specific osteoblastic genes in BMSCs pre-treated with LY294002 at the indicated time points. (D) Protein expression levels of Runx2 were determined by western blotting in the presence of CMS or LY294002 pre-treatment $48 \mathrm{~h}$ after CMS. (E) Runx2 expression levels were normalized to their respective total protein levels. Data were obtained from three individual experiments and presented as means \pm standard deviation. ${ }^{*} \mathrm{P}<0.05$ or ${ }^{* * *} \mathrm{P}<0.01$. BMSCs, bone mesenchymal stem cells; OVX BMSC, ovariectomized rat BMSC; CMS, continuous mechanical strain; ALP, alkaline phosphatase; Runx2, runt related transcription factor 2; COL I, type I collagen; con, control group.

The PI3K/Akt signaling pathway is key in the physiology and pathophysiology of various types of cell, exerting profound effects on processes, including proliferation, migration, metabolism and differentiation (29). In the current study, Akt was phosphorylated under the stimulation of CMS, with phosphorylation levels peaking at $15 \mathrm{~min}$ and then gradually declining; however, the level remained greater than that of the unloaded group. Meanwhile, as indicated by immunostaining, OVX BMSCs subjected to CMS demonstrated greater accumulation of p-Akt in the nucleus, indicating that mechanical strain enhances phosphorylation and nuclear translocation of the Akt protein. After confirming the activation of Akt, the OVX BMSCs were pre-treated with an inhibitor of the Akt signaling pathway (LY294002) to determine whether their strain-induced osteogenic commitment was dependent on Akt activation. Following treatment with LY294002, the strain-induced gene expression of osteogenic markers and Runx 2 protein expression decreased significantly. Previous studies demonstrated that Akt was particularly important in bone formation and was activated early in the transcriptional activation of osteogenesis $(15,30,31)$. Substantial evidence indicated that PI3K/Akt signaling was required for murine osteogenesis in vitro, including mouse embryonic fibroblasts, murine BMSCs, and in the mouse MSC line, C3H10T1/2 (17). Nuclear translocation of activated Akt may lead to the phosphorylation of key transcription factors, which in turn affects the levels of certain proliferation or differentiation-associated genes (32). Additionally, Akt is the mechanically activated kinase responsible for numerous other interventions. For example, the PI3K/Akt signaling pathway participates in 
matrix metalloproteinase- 2 expression by $10 \%$ mechanical stretch in vascular smooth muscle cells and by $18 \%$ in human aortic smooth muscle cells $(33,34)$. Furthermore, ultrasound stimulation promotes bone formation in osteoblasts via the integrin/protein tyrosine kinase 2/PI3K/Akt and extracellular-signal-regulated kinase signaling pathway (35). Studies also indicated that mammalian target of rapamycin complex 2 was required for mechanical activation of $\mathrm{Akt}$ and that mechanical inhibition of glycogen synthase kinase was dependent on Akt activation (36). However, as Akt is a pleiotropic signaling molecule with downstream targets that are differentially regulated depending upon the nature of the activating input, further studies investigating the downstream targets of strain-induced osteogenic commitment on Akt activation are required.

In conclusion, continuous short-term mechanical strain induced the early differentiation of OVX BMSCs towards an osteogenic phenotype, and CMS may activate the PI3K/Akt signaling pathway during osteoblastic differentiation. The present study may provide a promising strategy for regulating strain-induced bone remodeling in osteoporosis, however, further research is required regarding the downstream target and the in vivo conditions.

\section{Acknowledgements}

The present study was supported in part by grants from the National Natural Science Foundation of China (NSFC) (grant nos. 81371121, 11342005, 30901698, 10972142 and 81570950), the 'Chen Xing' Project from Shanghai Jiaotong University, and Shanghai Summit and Plateau Disciplines.

\section{References}

1. Diab DL and Watts NB: Postmenopausal osteoporosis. Curr Opin Endocrinol Diabetes Obes 20: 501-509, 2013.

2. Andreopoulou P and Bockman RS: Management of postmenopausal osteoporosis. Annu Rev Med 66: 329-342, 2015.

3. Kemmler W, Bebenek M, Kohl M and von Stengel S: Exercise and fractures in postmenopausal women. Final results of the controlled Erlangen fitness and osteoporosis prevention study (EFOPS). Osteoporos Int 26: 2491-2499, 2015.

4. Choi HJ: New antiresorptive therapies for postmenopausal osteoporosis. J Menopausal Med 21: 1-11, 2015.

5. Iwamoto J, Takeda T and Sato Y: Efficacy and safety of alendronate and risedronate for postmenopausal osteoporosis. Curr Med Res Opin 22: 919-928, 2006.

6. Appelman-Dijkstra NM and Papapoulos SE: Modulating bone resorption and bone formation in opposite directions in the treatment of postmenopausal osteoporosis. Drugs 75: 1049-1058, 2015.

7. Ehrlich PJ and Lanyon LE: Mechanical strain and bone cell function: A review. Osteoporos Int 13: 688-700, 2002.

8. Massafra U, Integlia D, Broccoli S and Migliore A: Mixed treatment comparison to rank antiresorptive agents in preventing new non vertebral fractures in postmenopausal osteoporosis Value Health 18: A636, 2015.

9. Weyts FA, Bosmans B, Niesing R, van Leeuwen JP and Weinans H: Mechanical control of human osteoblast apoptosis and proliferation in relation to differentiation. Calcif Tissue Int 72: 505-512, 2003.

10. Jagodzinski M, Drescher M, Zeichen J, Hankemeier S, Krettek C, Bosch U and van Griensven M: Effects of cyclic longitudinal mechanical strain and dexamethasone on osteogenic differentiation of human bone marrow stromal cells. Eur Cell Mater 7 : 35-41; discussion 41, 2004.

11. Liedert A, Kaspar D, Blakytny R, Claes L and Ignatius A: Signal transduction pathways involved in mechanotransduction in bone cells. Biochem Biophys Res Commun 349: 1-5, 2006.
12. Mauney JR, Sjostorm S, Blumberg J, Horan R, O'Leary JP, Vunjak-Novakovic G, Volloch V and Kaplan DL: Mechanical stimulation promotes osteogenic differentiation of human bone marrow stromal cells on 3-D partially demineralized bone scaffolds in vitro. Calcif Tissue Int 74: 458-468, 2004

13. Gao Y, Li JH, Han LC, Ma YQ, Hu J, Qu D and Xu YC: Osteoblastic differentiation and gene expression profile change in rat bone marrow mesenchymal stem cells after a single period of mechanical strain. Hua Xi Kou Qiang Yi Xue Za Zhi 27: 213-216, 2009.

14. Thompson WR, Rubin CT and Rubin J: Mechanical regulation of signaling pathways in bone. Gene 503: 179-193, 2012.

15. Baker N, Sohn J and Tuan RS: Promotion of human mesenchymal stem cell osteogenesis by PI3-kinase/Akt signaling, and the influence of caveolin-1/cholesterol homeostasis. Stem Cell Res Ther 6: 238, 2015.

16. Ghosh-Choudhury N, Abboud SL, Nishimura R, Celeste A, Mahimainathan L and Choudhury GG: Requirement of BMP-2-induced phosphatidylinositol 3-kinase and Akt serine/ threonine kinase in osteoblast differentiation and Smad-dependent BMP-2 gene transcription. J Biol Chem 277: 33361-33368, 2002.

17. Mukherjee A, Wilson EM and Rotwein P: Selective signaling by Akt 2 promotes bone morphogenetic protein 2-mediated osteoblast differentiation. Mol Cell Biol 30: 1018-1027, 2010.

18. Thompson DD, Simmons HA, Pirie CM and Ke HZ: FDA Guidelines and animal models for osteoporosis. Bone 17: 125S-133S, 1995.

19. Livak KJ and Schmittgen T: Analysis of relative gene expression data using real-time quantitative PCR and the $2-\Delta \Delta \mathrm{Ct}$ method. Methods 25: 402-408, 2001.

20. Marie PJ, Jones D, Vico L, Zallone A, Hinsenkamp M and Cancedda R: Osteobiology, strain, and microgravity: Part I. Studies at the cellular level. Calcif Tissue Int 67: 2-9, 2000.

21. Li R, Liang L, Dou Y, Huang Z, Mo H, Wang Y and Yu B: Mechanical strain regulates osteogenic and adipogenic differentiation of bone marrow mesenchymal stem cells. Biomed Res Int 2015: 873251, 2015.

22. Modder UI, Roforth MM, Hoey K, McCready LK, Peterson JM, Monroe DG, Oursler MJ and Khosla S: Effects of estrogen on osteoprogenitor cells and cytokines/bone-regulatory factors in postmenopausal women. Bone 49: 202-207, 2011.

23. Yamazaki S, Mizumoto T, Nasu A, Horii T, Otomo K, Denno H, Takebayashi T, Miyamoto K and Horiuchi T: Regulation of osteogenetic differentiation of mesenchymal stem cells by two axial rotational culture. J Artif Organs 14: 310-317, 2011.

24. Koike M, Shimokawa H, Kanno Z, Ohya K and Soma K: Effects of mechanical strain on proliferation and differentiation of bone marrow stromal cell line ST2. J Bone Miner Metab 23: 219-225, 2005.

25. Boeloni JN, de MON, Silva JF, Correa CR, Bertollo CM, Hell RC, de MPM, Goes AM and Serakides R: Osteogenic differentiation of bone marrow mesenchymal stem cells of ovariectomized and non-ovariectomized female rats with thyroid dysfunction. Pathol Res Pract 209: 44-51, 2013.

26. Varkey M, Kucharski C, Doschak MR, Winn SR, Brochmann EJ, Murray S, Matyas JR, Zernicke RF and Uludag H: Osteogenic response of bone marrow stromal cells from normal and ovariectomized rats treated with a low dose of basic fibroblast growth factor. Tissue Eng 13: 809-817, 2007.

27. Wu Y, Zhang P, Dai Q, Yang X, Fu R, Jiang L and Fang B: Effect of mechanical stretch on the proliferation and differentiation of BMSCs from ovariectomized rats. Mol Cell Biochem 382: 273-282, 2013.

28. Zhang P, Dai Q, Ouyang N, Yang X, Wang J, Zhou S, He N, Fang B and Jiang L: Mechanical strain promotes osteogenesis of BMSCs from ovariectomized rats via the ERK1/2 but not $\mathrm{p} 38$ or JNK-MAPK signaling pathways. Curr Mol Med 15: 780-789, 2015.

29. Ping C, Lin Z, Jiming D, Jin Z, Ying L, Shigang D, Hongtao Y, Yongwei $\mathrm{H}$ and Jiahong D: The phosphoinositide 3-kinase/ Akt-signal pathway mediates proliferation and secretory function of hepatic sinusoidal endothelial cells in rats after partial hepatectomy. Biochem Biophys Res Commun 342: 887-893, 2006.

30. Tsai KS, Kao SY, Wang CY, Wang YJ, Wang JP and Hung SC: Type I collagen promotes proliferation and osteogenesis of human mesenchymal stem cells via activation of ERK and Akt pathways. J Biomed Mater Res A 94: 673-682, 2010.

31. Ling L, Dombrowski C, Foong KM, Haupt LM, Stein GS, Nurcombe V, van Wijnen AJ and Cool SM: Synergism between Wnt3a and heparin enhances osteogenesis via a phosphoinositide 3-kinase/Akt/RUNX2 pathway. J Biol Chem 285: 26233-26244, 2010 . 
32. Das M, Bouchey DM, Moore MJ, Hopkins DC, Nemenoff RA and Stenmark KR: Hypoxia-induced proliferative response of vascular adventitial fibroblasts is dependent on g protein-mediated activation of mitogen-activated protein kinases. J Biol Chem 276: 15631-15640, 2001

33. Seo KW, Lee SJ, Kim YH, Bae JU, Park SY, Bae SS and Kim CD: Mechanical stretch increases MMP-2 production in vascular smooth muscle cells via activation of PDGFR-beta/Akt signaling pathway. PLoS One 8: e70437, 2013.

34. Liu X, Huang X, Chen L, Zhang Y, Li M, Wang L, Ge C, Wang H and Zhang M: Mechanical stretch promotes matrix metalloproteinase-2 and prolyl-4-hydroxylase alphal production in human aortic smooth muscle cells via Akt-p38 MAPK-JNK signaling. Int J Biochem Cell Biol 62: 15-23, 2015.
35. Tang CH, Yang RS, Huang TH, Lu DY, Chuang WJ, Huang TF and Fu WM: Ultrasound stimulates cyclooxygenase-2 expression and increases bone formation through integrin, focal adhesion kinase, phosphatidylinositol 3-kinase, and Akt pathway in osteoblasts. Mol Pharmacol 69: 2047-2057, 2006.

36. Case N, Thomas J, Sen B, Styner M, Xie Z, Galior K and Rubin J: Mechanical regulation of glycogen synthase kinase 3beta (GSK3beta) in mesenchymal stem cells is dependent on Akt protein serine 473 phosphorylation via mTORC2 protein. J Biol Chem 286: 39450-39456, 2011. 